\title{
Sevrapek City ou la courte histoire du groupe de recherche "Forêts-Montagnes-Rivières » (expédition Santo 2006, Vanuatu)
}

\section{Fabienne Tzérikiantz}

\section{(2) OpenEdition Journals}

Édition électronique

URL : http://journals.openedition.org/jso/3602

DOI : $10.4000 /$ jso.3602

ISSN : $1760-7256$

Éditeur

Société des océanistes

Édition imprimée

Date de publication : 15 décembre 2008

Pagination : 207-220

ISBN : 978-2-85430-012-3

ISSN : 0300-953x

Référence électronique

Fabienne Tzérikiantz, "Sevrapek City ou la courte histoire du groupe de recherche « Forêts-MontagnesRivières » (expédition Santo 2006, Vanuatu) », Journal de la Société des Océanistes [En ligne], 126-127 | Année 2008, mis en ligne le 15 décembre 2011, consulté le 20 juin 2020. URL : http:// journals.openedition.org/jso/3602 ; DOI : https://doi.org/10.4000/jso.3602 


\title{
Sevrapek City ou la courte histoire du groupe de recherche " forêts-montagnes-rivières » (expédition Santo 2006, Vanuatu)
}

par

\author{
Fabienne TZÉRIKIANTZ*
}

\section{RÉSUMÉ}

D'août à décembre 2006, un inventaire de la biodiversité "sans précédent" s'est tenu sur l'île de Santo, au nord de l'archipel du Vanuatu. Cette aventure scientifique "d'exception" mettant en scène des "Indiana Jones de la biodiversité " sur une île certes peuplée mais " manifestement sous-explorée » a semble-t-il rassemblé tous les ingrédients nécessaires à un déchaînement médiatique. Conduisant des enquêtes ethnologiques sur la très isolée côte ouest de Santo depuis 1996, j'ai été sollicitée pour participer au groupe "forêtsmontagnes-rivières » (le " module FMR ») afin d'étudier la perception qu'ont eue les populations locales du nordouest de Santo de cet événement inhabituel. C'est là qu'était établi un camp de base construit par les habitants de la région pour accueillir, pendant plus d'un mois, chercheurs, équipes de tournage, photographes, journalistes, organisateurs du projet et travailleurs recrutés dans la région. Une fois l'expédition terminée, j'ai poursuivi mon travail de décembre à mars 2007, en conduisant mes enquêtes hors du cadre de l'expédition et en co-réalisant un film documentaire sur l'après-Santo 2006 (intitulé Sevrapek City) avec les villageois du nord-ouest de Santo et certains représentants des autorités locales, tous impliqués à un moment ou à un autre dans le déroulement de cette mission scientifique. Dans cet article, les résultats des recherches que j’ai menées pendant et après l'expédition soulèvent plusieurs questions : l'ambition éthique de la mission, si chère à ses organisateurs, a-t-elle été atteinte? Qu'est-il advenu des promesses concernant les conditions d'implication des partenaires locaux? De quel " partage des avantages" parle-t-on? Quelle restitution pour les spécimens collectés au Vanuatu?

Mots-ClÉs : Vanuatu, Santo, perception locale, expédition Santo 2006, médiatisation, questions éthiques

\begin{abstract}
From August to December 2006, a large inventory of biodiversity took place on Santo, a small island in Northern Vanuatu. A bunch of " savages » and a big mob of "Indiana Jones " roaming an " unexplored country " were perfect ingredients for media enthusiasm. Because I had been conducting fieldwork in the remote West Coast of Santo for twelve years, I have been asked to become a member of the "Forests-Mountains-Rivers" (FMR) team of this expedition, in order to evaluate the impact of this project on the local communities of this area. From December to March 2007, I kept on conducting researches on this topic out of the frame of Santo 2006 when the expedition ended. At this stage, I got involved into the shooting of a documentary movie that depicts this very situation, entitled Sevrapek City. We interviewed both local authorities and the inhabitants who were involved in some way or another with the expedition. Santo 2006 rises up numerous questions and debates. In this paper are presented various critics made both by local people and local authorities. They reflect the contrast between what was expected and what really happened. The remarks concerned the involvement of local institutions (such as the Cultural Centre of Vanuatu and its fieldworkers), the involvement of local people from rural area, the ethical aspects of Santo 2006 concerning the handling of local workers and their wages and Santo 2006 commitment concerning the repatriation of all biota collected.
\end{abstract}

KeYwords: Vanuatu, Santo, local perception, Santo 2006 expedition, media coverage, ethical issues

* CREDo CNRs, université de Provence, Marseille, tz-fab@club-internet.fr 
D'août à décembre 2006, l'île de Santo, au nord de l'archipel du Vanuatu (carte 1), a accueilli les membres de l'expédition naturaliste Santo 2006 au nombre desquels plus de cent cinquante chercheurs venus y conduire un inventaire de la biodiversité. Cette «fabuleuse aventure scientifique » s'est achevée avec « un grand succès », comme l'ont annoncé les nombreux articles parus dans la presse en 2006 et 2007. Par son ambition scientifique (découvrir en un temps record de nouvelles espèces et inventorier la biodiversité afin d'évaluer l'ampleur et les conséquences de sa perte, anticipée dans l'avenir), cette expédition one shot ${ }^{1}$ aux moyens financiers et logistiques considérables a employé des dizaines de scientifiques et spécialistes de nationalités et disciplines différentes ${ }^{2}$ et eu recours à des technologies parmi les plus novatrices, les plus coûteuses et les plus spectaculaires qui soient.

$\mathrm{Ni}$ les attentes scientifiques de ses organisateurs ni celles des financeurs (fonds publics, mais surtout fonds privés), encore moins celles du pays hôte ou celles de la cohorte de médias (radios, journaux télévisés, presse quotidienne, magazines grand public, reportage de 110 minutes pour le magazine Thalassa...) sollicités sans économie pour couvrir cet événement "planétaire », ne devaient être déçues. Autrement dit, si l'originalité de Santo 2006 résidait dans une volonté d'exploration la plus exhaustive possible de la biodiversité de Santo et dans un déploiement de moyens exceptionnels, tant au plan financier qu'humain et matériel ${ }^{3}$, on l'aura compris, la particularité de cette mission scientifique résidait aussi dans son extrême médiatisation, voire sa mise en spectacle.

\section{Santo 2006 et l'île de Santo}

Rappelons que l'île de Santo, malgré sa longue histoire de rencontres et de confrontations avec l'Occident, et bien qu'étant l'un des endroits où se formèrent des mouvements identitaires qui conduisirent les Nouvelles-Hébrides, cet ancien condominium franco-britannique, à l'indépendance en 1980, continue de véhiculer une image fixe, comme hors du temps. Elle demeure une

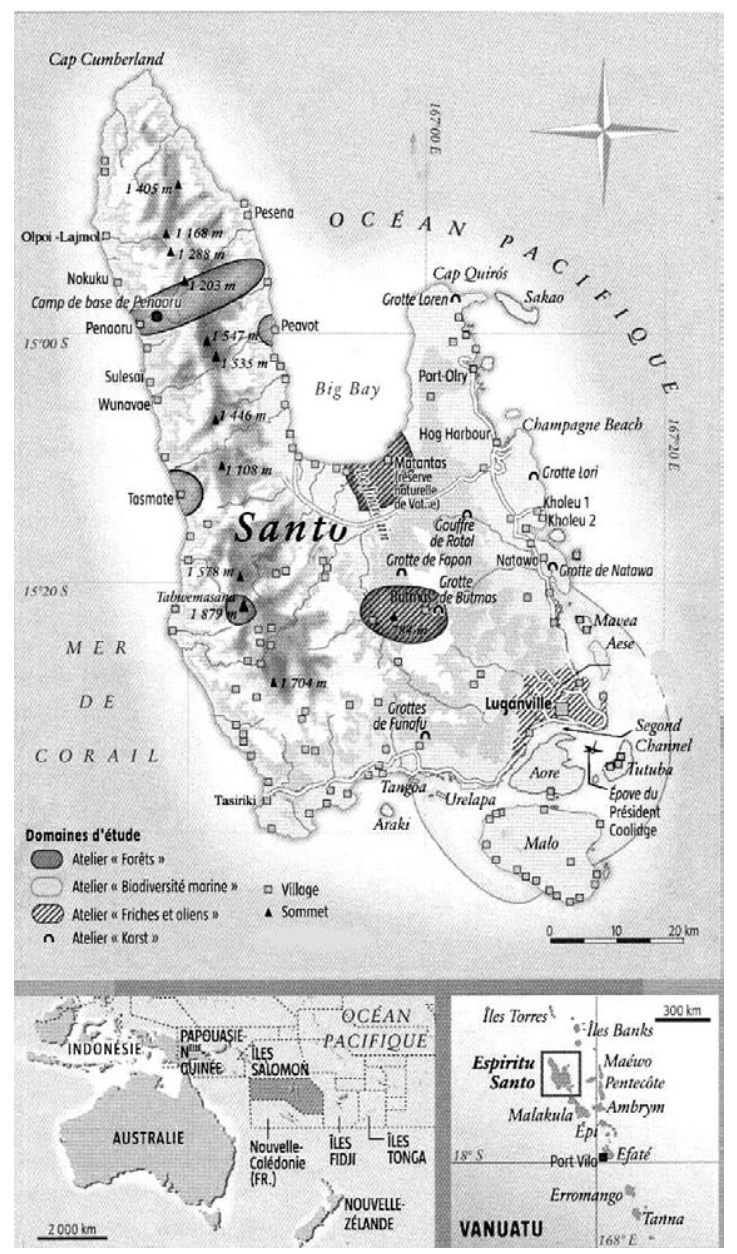

CARTE 1. - Carte de localisation (d'après Tardieu et Barnéoud, $2007: 9)$

« île aux trésors » (Lescroart, $2007: 13$ ) dotée d'une extrême diversité culturelle, écologique et linguistique, quasiment inexplorée, et regorgeant de curiosités aussi bien biologiques (espèces inconnues) qu'humaines (peuples non contactés vivant en adéquation parfaite avec un environnement luxuriant). Voilà ce que, sans relâche, journalistes, photographes, aventuriers et documentaristes venus du monde entier espèrent encore aujourd'hui trouver à Santo.

Même si ce n'était pas ce qui attirait officiellement ceux venus couvrir l'événement scientifique en question, Santo 2006 avait tout pour séduire : des « Indiana Jones de la biodiversité »

1. Ce terme, signifiant littéralement « un tir » ou « un coup », a été employé lors d'une réunion de travail en juin 2006 par Philippe Bouchet (malacologue du Muséum national d'histoire naturelle et initiateur de cette expédition). Il entendait par là que cette mission scientifique n'aurait a priori pas de suite au Vanuatu, mais aussi qu'une expédition comparable pourrait être organisée ailleurs dans le monde, en fonction de ses résultats.

2. Entomologistes, botanistes, malacologues, mycologues, herpétologistes, ichtyologues, spéléologues, archéologues, ethnologues... mais aussi plongeurs et grimpeurs-élagueurs professionnels, cuisiniers et comptables.

3. Je fais ici allusion à un navire océanographique mis à disposition par l'Institut de recherche pour le développement (IRD) et au nouveau prototype du Radeau des Cimes de l'ONG Pro-Natura International, qui, associée au MNHN et à l'IRD, faisait partie du consortium organisateur de l'expédition. 


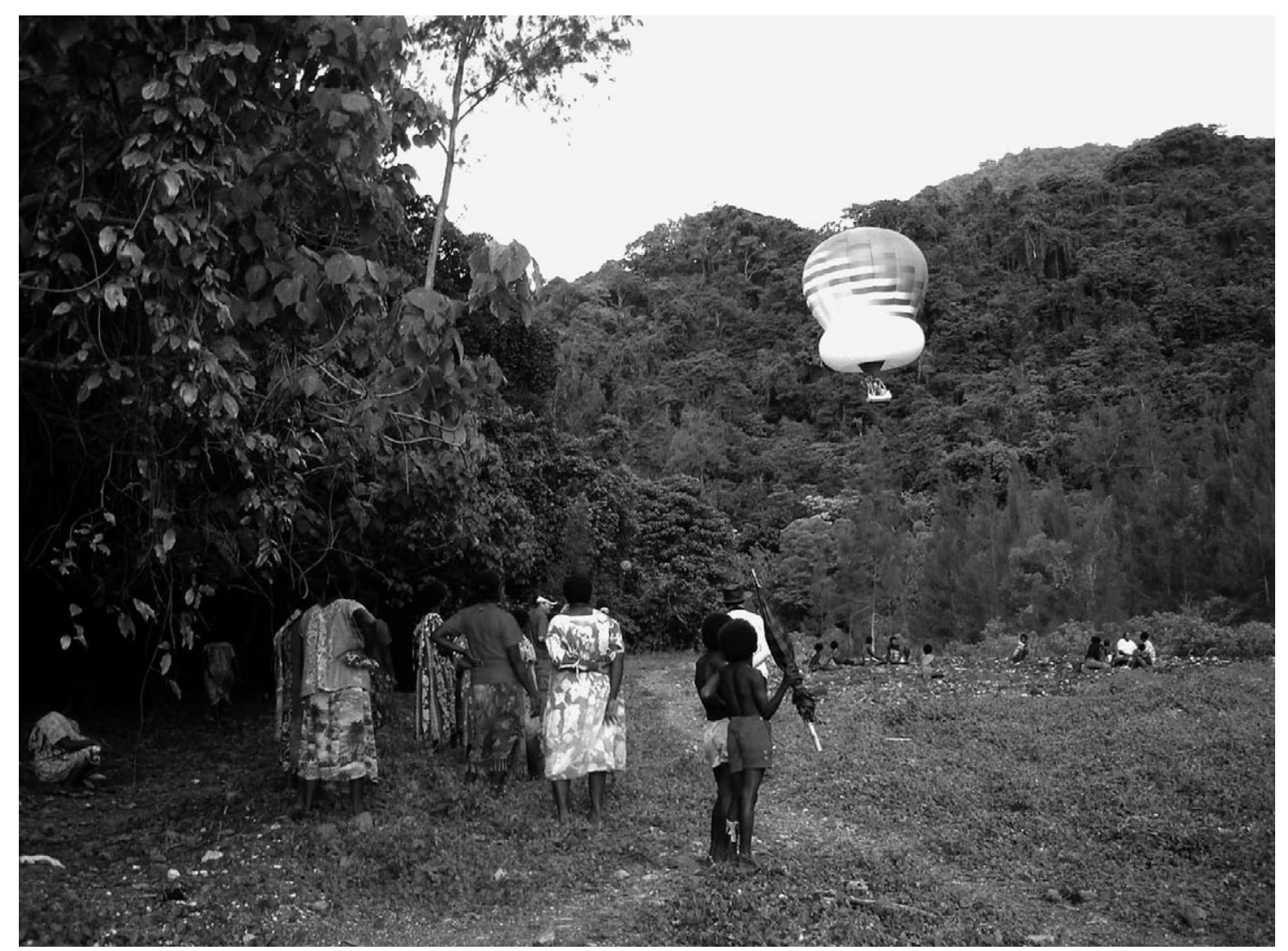

Рното 1. - L'arboglisseur (cliché de l'auteur)

(Collomb, 2007: 122-123) ou « savanturiers » (Schmitt, 2007 : 52), passionnés et couverts de boue, arpentant résolument cette île de la cime des arbres aux fonds marins et s'émerveillant à la vue d'insectes et de mollusques particulièrement photogéniques, une île «mystérieuse » offrant autant de forêts tropicales montagneuses que de plages coralliennes, enfin, une population majoritairement rurale d'agriculteurs et de pêcheurs qui, bien que vivant dans un jeune État décolonisé depuis bientôt trente ans et étant impliqués dans l'économie de marché et dans la politique de leur pays depuis près d'un siècle, continuent de valoriser leurs pratiques et savoirs coutumiers dans de petites maisons de bambou. C'est pour partie le mode de vie apparemment inchangé de ces gens qui continue, malgré leur entrée de longue date dans le "monde moderne », de favoriser la diffusion pléthorique de clichés à leur égard.

Autant que les fantasmes suscités par les stéréotypes d'une aventure scientifique prenant place dans une telle contrée, la présence d'un arboglisseur ${ }^{4}$, un aérostat installé de surcroît tout près d'un petit village très isolé du nordouest de l'île de Santo, allait attirer trois équipes de tournage, quatre journalistes, plusieurs photographes... et motiver la venue d'une délégation officielle composée de ministres du Vanuatu et de représentants des autorités françaises, mais aussi des sponsors de cet appareil qui, en fait, passa la plus grande partie de son temps au sol (photo 1).

Bien qu'ayant tenté d'échapper à ces stéréotypes, le document officiel de l'expédition Santo 2006 affirmait :

« Santo (ou Espiritu Santo) est la plus grande île du Vanuatu : $3677 \mathrm{~km}^{2}$, trois fois Tahiti, la moitié de la Corse, pour seulement 30000 habitants et... une quarantaine de langues !! Santo est aussi l'île la plus élevée de tout l'archipel [...]. Les richesses naturelles sont résumées par ce commentaire lapidaire du guide Lonely Planet: "Sparkling blue holes, unlogged rainforests and the world's largest accessible shipwreck". Compte tenu de sa superficie, de son relief et de son âge géologique (Miocène), Santo est manifestement sous-explorée et de nombreuses découvertes sont à attendre dans tous les compartiments de la biodiversité. » (Bouchet et al., 2006 : 3)

4. Il s'agit du nouveau prototype du Radeau des Cimes de Pro-Natura. Il est constitué d'une sorte de plate-forme suspendue sous un ballon pouvant se poser sur la canopée et conçue pour permettre aux scientifiques de collecter, dans des conditions inédites, insectes, fruits et fleurs. 
De l'avis de tous (organisateurs, médias, chercheurs), les ambitions de Santo 2006 ont été atteintes : un état des lieux de la biodiversité a été réalisé en très peu de temps et l'on pense que sur les dix mille espèces collectées, plus de mille espèces nouvelles ont été découvertes (Bouchet et al., 2007). Comme le dit Philippe Bouchet, malacologue du Muséum national d'histoire naturelle de Paris, initiateur et co-organisateur de ce projet, je cite :

« Les résultats [sont] à la hauteur de nos espérances $[\ldots] »($ Barrais, 2007)

Mais, d'après le document officiel de l'expédition (Bouchet et al., 2006), l'ambition de Santo 2006 était autant scientifique qu'éthique :

«L'opération Santo se veut exemplaire dans le cadre des obligations éthiques et politiques d'un pays du Nord (la France) vis-à-vis d'un pays du Sud, ancienne colonie [...] à la fin de l'expédition, l'équipe du projet remettra au gouvernement du Vanuatu des collections de spécimens biologiques, en particulier, des plantes, des poissons, des vertébrés fossiles et des insectes [...]. Une attention particulière sera apportée à l'implication des partenaires locaux, à la restitution de l'information pour le développement durable, à l'éducation environnementale. » (Bouchet et al., 2006 : 9)

Qu'en a-t-il été de ces ambitions et promesses ? Les résultats de Santo 2006 sont-ils aussi à la hauteur des espérances des populations et des autorités locales du Vanuatu?

Conduisant des enquêtes ethnologiques sur la côte ouest de Santo depuis 1996, j'ai été sollicitée pour participer au groupe « Forêts-MontagnesRivières $^{5} »$ (le « module FMR ») afin d'étudier la perception qu'ont eue les populations locales de cet événement exceptionnel et de mesurer l'impact du projet sur les populations du nordouest de Santo (voir carte 1). C'est dans cette région de l'île qu'était établi un camp de vie pour les scientifiques, localement nommé Sevrapek (le « ruisseau de l'arbre à pain »), construit par les habitants de la région pour accueillir, pendant plus d'un mois, chercheurs, équipes de tournage, photographes, journalistes, organisateurs du projet et travailleurs locaux. Une fois l'expédition terminée, j'ai poursuivi mes enquêtes de décembre à mars 2007, hors du cadre de l'expé- dition ${ }^{6}$ et en co-réalisant un film documentaire sur l'après-Santo 2006 (intitulé Sevrapek City) ${ }^{7}$ dans lequel les villageois du nord-ouest de Santo et certains représentants des autorités locales ces fameux "partenaires locaux » impliqués à un moment ou à un autre dans le déroulement de cette mission - ont livré leurs sentiments (et ressentiments) sur la conduite de ce projet scientifique. Cet article est donc le fruit des recherches que j'ai menées pendant et après Santo 2006.

Une fois évoqués succinctement le montage de l'expédition et les négociations menées entre les organisateurs de Santo 2006 et les autorités locales, je me pencherai plus particulièrement sur le groupe de recherche « Forêts Montagnes Rivières ", à propos duquel seront exposés le déroulement des négociations avec les populations du nord-ouest de Santo, la construction du camp, les relations entre les participants de la mission scientifique et les villageois, pour aboutir enfin à ce qui fut l'objectif des enquêtes que j'ai menées une fois la vague passée : la perception locale de cet événement et de la désertion du camp, un mois plus tard.

\section{L'organisation de l'opération}

Santo 2006 est le fruit d'une rencontre entre deux institutions françaises de recherche de renom (le MNHN avec Philippe Bouchet, et l'IRD avec Hervé le Guyader) et une organisation non gouvernementale (ONG), Pro-Natura International (représentée par Olivier Pascal). C'est pour faire face aux problèmes financiers touchant certaines disciplines « pauvres » du MNHN, comme l'entomologie, mais aussi dans le but de réaliser une expédition one shot susceptible d'être ensuite appliquée ailleurs ${ }^{8}$, que Philippe Bouchet, habitué à monter de grandes expéditions sur la biodiversité marine (comme en 2004 aux Philippines), a décidé de s'associer à cette ONG.

Mais qu'est-ce que Pro-Natura? Familiarisée depuis une dizaine d'années avec le montage de grandes expéditions sur la canopée des forêts tropicales (autour de Bruno Corbara, d'Olivier Pascal et de Dany Cleyet-Marrel, l'équipe du Radeau des Cimes/Pro-Natura-Ibisca), cette ONG a par ailleurs officiellement pour ambition

5. Les recherches conduites durant l'expédition ont concerné tous les compartiments de la biodiversité (grands fonds marins, récifs, grottes, eaux douces, montagnes, canopées forestières) de Santo. La recherche a ainsi été organisée en quatre "modules » naturalistes (« Biodiversité marine »; « Karst (et grottes) »; « Friches et aliens »; «Forêts, montagnes et rivières ») et un module de sciences sociales (« Perceptions plurielles de la biodiversité »).

6. Grâce à un financement du CREDo et de la Fondation de la Maison des sciences de l'homme.

7. Avec Emmanuel Broto, opérateur de prises de vue.

8. Suite au succès de Santo 2006, d'autres missions d'inventaire de la biodiversité sur les hot spots de la biodiversité mondiale sont actuellement en préparation. Se reporter au site de Pro-Natura International (rubrique « biodiversité » puis " Planète revisitée »). 
de « répondre aux problèmes socioéconomiques et environnementaux des communautés rurales des pays du Sud» ( http://www.pronatura.org). Elle a surtout permis à l'expédition Santo 2006 de bénéficier de ses réseaux de financements privés ${ }^{9}$, d'innovations technologiques particulièrement adaptées à la médiatisation, comme l'arboglisseur, ce nouveau prototype du Radeau des cimes, mais aussi de son expérience en termes de communication. Pro-Natura est en effet affiliée de façon exclusive à l'agence de Communication Médias-Multi-médias Aтом Productions (associée à MVC Productions dans le cadre de la production audiovisuelle d'un film de 110 minutes pour le magazine Thalassa) qui, dans le cadre de Santo 2006, s'est affiliée à l'agence Gamma. L'association du MNHN et de l'IRD avec ProNatura a conféré de fait une dimension spectaculaire (et critiquable) à cette expédition qui a ainsi bénéficié d'un budget de 1,2 millions d'euros ${ }^{10}$. Le fait de bénéficier majoritairement de fonds privés émanant de mécènes mesurant leur retour sur investissement en terme de retour d'images, impliquant d'accorder le monopole exclusif des images produites dans le cadre de Santo 2006 à une société de communication $(\text { АTOM })^{11}$, pose finalement la question des conséquences d'un financement de type privé sur les missions scientifiques portées par des institutions publiques.

Par ailleurs, il faut ici évoquer ce qui a poussé Philippe Bouchet à choisir le Vanuatu : ses raisons relèvent d'un compromis entre un intérêt scientifique pour l'île de Santo et les facilités politico-administratives supposées de ce petit État insulaire du Pacifique Sud ${ }^{12}$. En effet, bien qu'investi dans ses droits culturels avec son très actif Centre culturel, le Vanuatu ne possède aucune structure pour contrôler efficacement des recherches biologiques étrangères et pour en négocier les contreparties ${ }^{13}$. Mais lors des deux missions de prise de contact avec les autorités politiques du Vanuatu (en mars et octobre 2005), les organisateurs prirent conscience, au moment de signer la convention (Memorandum of Understanding) entre le consortium MNHN-IRDPro-Natura, le gouvernement du Vanuatu, son Unité environnementale et le Centre culturel du Vanuatu à Port-Vila, que, malgré l'apparente facilité des démarches politiques et administratives, certaines résistances inattendues se faisaient jour. Officiellement, ces difficultés ont été résolues bien que, de mon point de vue, les demandes locales, pourtant clairement formulées par les institutions ni-vanuatu en présence lors de l'élaboration de cette convention, n'aient reçu qu'un faible écho - voire un écho de principe seulement puisque, comme on le verra, elles n'ont pas été respectées dans les faits - et aient surtout servi les intérêts de l'expédition. Élaborée par les organisateurs de Santo 2006 et reformulée après les négociations que je vais brièvement rappeler, cette convention a finalement été signée le 24 mars 2006

Dès mars 2005, plusieurs réunions de préparation se sont donc tenues, en France et au Vanuatu, pour structurer le programme scientifique, les questions financières et pour tenter d'apporter une réponse adaptée à la question : «Que va-t-on laisser au Vanuatu? ». Des difficultés apparurent en octobre 2005 à l'occasion d'une rencontre avec Donna Kalfatak, membre des services de l'environnement et spécialiste de la faune d'eau douce, par ailleurs habituée à travailler avec des chercheurs du MNHN comme l'ichtyologue Philippe Keith (membre de l'expédition Santo 2006). La première critique concernait l'exportation des spécimens récoltés par les biologistes : il fallait laisser les échantillons au Vanuatu. Aussi Donna Kalfatak demanda-t-elle qu'un doublon des spécimens déjà connus (par les scientifiques) soit laissé au Vanuatu, les autres devant être renvoyés au Vanuatu une fois identifiés. Le gouvernement réclama ensuite que soit dressée une liste de tous les résultats des recherches antérieures menées au Vanuatu, ainsi qu'une liste des spécimens conservés au sein des différentes institutions françaises. Voici le

9. Fondation Niarchos $(500000 €)$; Fondation Total $(150000 €)$; Nature et Découvertes $(15000 €)$; Solvay-Solvin pour « l'arbodôme » (une tente de protection abritant la montgolfière) et Ricoh pour l'arboglisseur (400 $000 €$ ). Se reporter au site www.Santo2006.org.

10. Hors salaires des chercheurs et mise à disposition d'un navire océanographique par l'IRD.

11. Voici un extrait des « conditions générales » de la « fiche participant et/ou visiteur » signée par tous les intervenants sur la mission : « Le participant [...] déclare être informé que les droits de reproduction ou de représentation de toute photographie ou prise de vue réalisée sur le ou les sites de la mission "Santo 2006" ont été réservés par les co-Organisateurs ; en conséquence, il s'interdit de communiquer aucune photographie ou prise de vues effectuées par lui ou par un tiers à quelque organisme de presse ou d'édition ou de reproduction que ce soit et ou d'en céder les droits à quiconque, que ce soit pour une publication ou une représentation sur quelque support que ce soit, sauf autorisation expresse des co-Organisateurs ».

12. Elsa Faugère (INRA-SAD, Écodéveloppement, Avignon), « Anthropologie de la biodiversité : l'expédition Santo 2006. Premiers éléments », séminaire du 23 juin 2006, INRA, Montpellier.

13. «On n’a pas de système adéquat pour légiférer. Le Centre culturel du Vanuatu n'est pas fait pour ça et on n'est pas préparé au niveau gouvernemental pour contrôler ce genre de recherches sur les ressources biologiques [...] » (Ralph Regenvanu, Directeur du Vanuatu National Cultural Council [VNCC], mars 2007, Port-Vila). Voir aussi Royson, 2006. 
contenu de l'article 2 de la convention signée en mars 2006, une fois qu'eut été pris en compte le résultat de ces négociations :

«Le Muséum s'engage à rendre au Vanuatu les collections de spécimens biologiques collectés durant le projet. En particulier les plantes (département des Forêts), les poissons (départements des Pêches), les fossiles vertébrés (Centre culturel), les insectes et autres biotes [tout être vivant] (Quarantaine) et tout autre biote dans des lieux à préciser. »

Sans qu'aucune liste préalable ait été établie ${ }^{14}$, empêchant donc, de facto, de savoir si l'intégralité des collections de spécimens serait restituée au Vanuatu, la convention engageait donc le projet à restituer ces échantillons dans les embryons de collections y existant déjà. Il n'est pas inutile de rappeler ici que les institutions nationales désignées ne sont nullement équipées pour conserver d'aussi importantes collections dans de bonnes conditions (manque de place, de moyens et de personnel). Un tel engagement émanant du MNHN, qui n'impliquait nullement la construction d'infrastructures adaptées, peut donc laisser perplexe.

La deuxième réserve émise par Donna Kalfatak et par le directeur du Centre culturel du Vanuatu (à l'époque Ralph Regenvanu ${ }^{15}$ ) concernait les risques d'utilisation commerciale des spécimens. Malgré un certain vide législatif concernant le contrôle des recherches étrangères sur les ressources naturelles (note 13), le gouvernement du Vanuatu demanda aux organisateurs du projet de connaître l'identité des financeurs privés de l'expédition. Il voulait savoir si y figuraient des firmes pharmaceutiques ou d'autres sociétés commerciales, ce qui aurait nécessité la signature de conventions particulières. Dans l'article 2 de la convention, il fut spécifié que les participants de l'expédition ne collecteraient leurs informations et leurs spécimens que dans un but académique, c'est-à-dire non commercial ou industriel.

La troisième critique portait sur le statut des savoirs locaux, c'est-à-dire sur l'absence de recours au Centre culturel de Port-Vila et à ses fieldworkers (des enquêteurs de terrain volontai- res formés par ce centre depuis la fin des années 1970) ${ }^{16}$. Était aussi déplorée l'absence de la notion de "consentement informé préalable» nécessaire avant toute collecte de savoirs locaux sur la biodiversité. Aussi, dans l'article 4 de la convention, fut ajouté le paragraphe suivant :

« Le Muséum s'engage à impliquer les enquêteurs de terrain du Centre culturel et à employer des travailleurs issus des communautés [...]. Les données recueillies dans le cadre de l'expédition sont la propriété intellectuelle du gouvernement du Vanuatu sauf dans le cas où les données constituent un savoir traditionnel ou indigène, auquel cas, ces données restent la propriété intellectuelle de l'(des) informateur(s). ${ }^{17}$ »

Quant aux organisateurs de Santo 2006, ils ont obtenu du gouvernement du Vanuatu des facilités pour l'obtention des visas de recherche de tous les participants, des facilités pour faire entrer au pays les équipements nécessaires, mais aussi pour faire sortir tous les spécimens « liés au projet ou en résultant » (article 3 de la convention). Il a par ailleurs été demandé au gouvernement d'offrir les meilleures conditions possibles en termes de détaxe pour les membres de l'expédition (voiture, matériel divers, essence).

\section{Le module « Forêts-Montagnes-Rivières » sur la côte ouest de Santo}

La mission que je devais remplir au sein de l'expédition Santo 2006 s'est déroulée en novembre 2006 au sein du " module FMR », soit dans le lieu de vie des scientifiques, sorte d'étrange hameau construit au milieu de la forêt par la main-d'œuvre locale à $4 \mathrm{~km}$ du petit village littoral de Penaoru (106 habitants à ce moment-là ; photo 2) et à quelques centaines de mètres du site de l'arboglisseur. Ce groupe de recherche, comprenant une soixantaine de chercheurs spécialisés dans la faune et la flore terrestre et d'eau douce, était placé sous la responsabilité d'Olivier Pascal (Pro-Natura International). C'est à cet endroit que le déchaînement médiatique a été le plus visible : plusieurs journalistes et photographes, ainsi que les trois équipes de tournage de la

14. «Ils ont dit qu'ils renverraient les échantillons de tout [...]. Je n'y crois pas. Premièrement, on ne sait pas exactement ce qu'ils ont emporté, il n'existe aucune liste ! Alors comment savoir si ce qu'ils restituent correspond à ce qu'ils ont pris ? [...] Deuxièmement : que vont-ils en faire ? On ne sait pas... Quand ça sort du pays, on ne sait pas ce qui se passe... [...] » (Marcellin Abong, directeur du Centre culturel du Vanuatu, mars 2007, Port-Vila).

15. Remplacé fin 2006 par Marcellin Abong, Ralph Regenvanu a ensuite pris le poste de directeur du Vanuatu National Cultural Council [vNCC].

16. Se reporter au Cultural Research Policy du Centre culturel : www.vanuatuculture.org/research/050520_cultural researchpolicy.html.

17. Ce dernier point s'appuie sur l'un des articles du Cultural Research Policy du Centre culturel qui stipule ceci : « Kastom [terme qui en bislama, la langue véhiculaire du Vanuatu, se traduit par coutume ou tradition] belongs to individuals, families, lineages and communities in Vanuatu. Any research on kastom must, in the first instance, respond to and respect the needs and desires of those people to whom the kastom belongs. " 


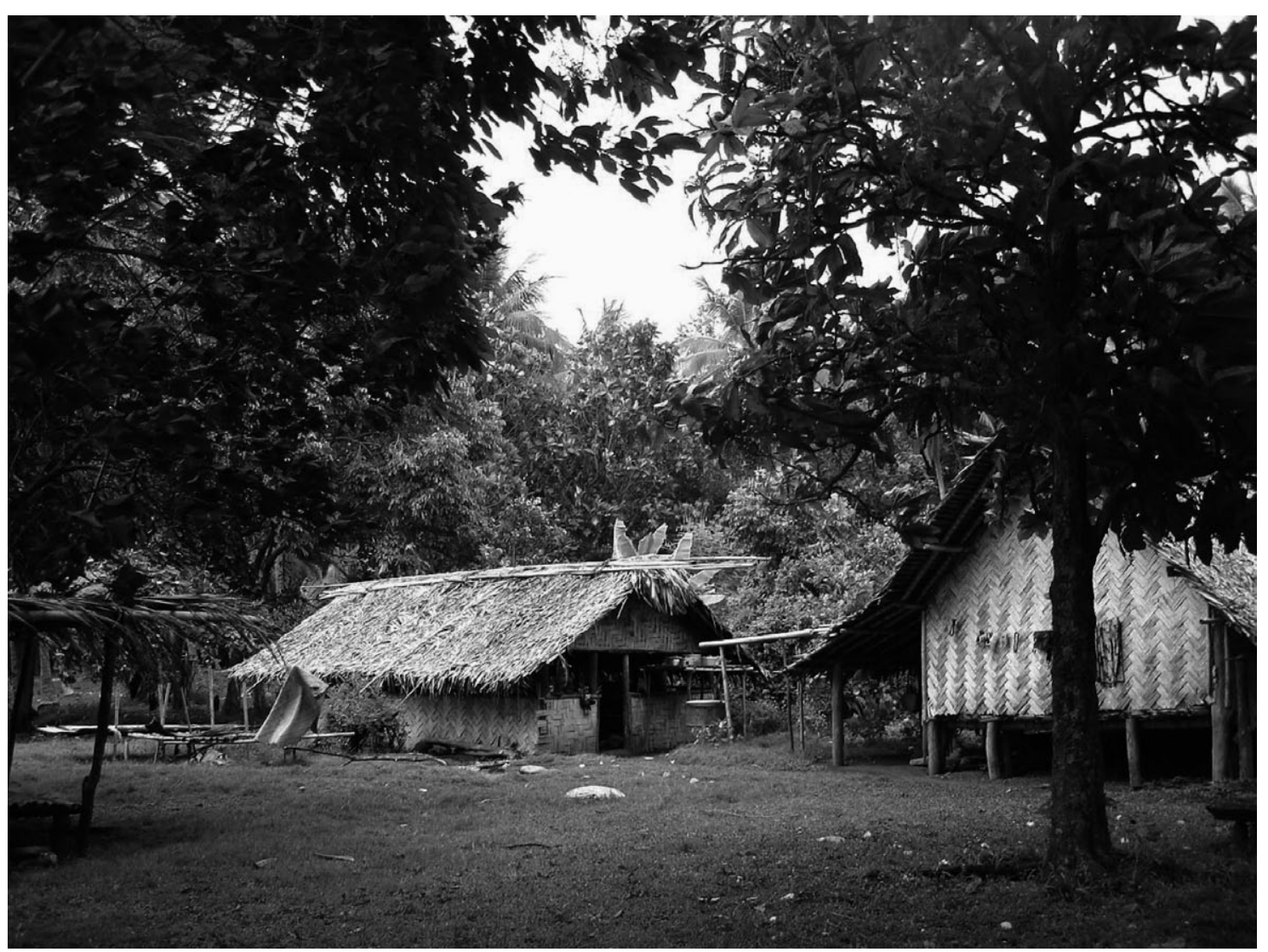

Photo 2. - Le village de Penaoru (cliché de l'auteur)

société ATOM/MVC - qui détenait, on l'a dit, le monopole exclusif des images produites pendant l'expédition et sur ce site - étaient présents pour couvrir l'événement, qu'il s'agisse de la vie des scientifiques au camp, de leur travail dans les laboratoires et dans les différents campements établis en altitude (entre 900 et 1200 mètres) ou des multiples (et souvent vaines) tentatives de vol de l'arboglisseur.

Pour comprendre ce qu'a pu représenter pour les gens de l'Ouest de Santo la venue de ces dizaines de chercheurs occidentaux, mais aussi un tel déploiement de haute technologie et une telle abondance de biens matériels, il est nécessaire de présenter rapidement cette partie de l'île. $\mathrm{Si}$ sa côte orientale attire de nombreux touristes par son accès facile, ses plateaux coralliens et ses plages de sable blanc, en revanche, la morphologie accidentée du paysage de sa partie occidentale, ses forêts très denses, ses rivières tumultueuses et la faible densité de sa population, ajoutés à l'absence de routes et à un fort taux de paludisme, rendent cette région plutôt inhospitalière. Elle présente cependant des attraits pour les visiteurs étrangers, car si les touristes y sont rares, elle suscite depuis plus d'un siècle l'intérêt de commerçants (bois de santal ou de kaori - Aga- this macrophylla -, minerais métallifères divers), et la curiosité d'aventuriers en tout genre et de naturalistes. S'il existe bien une piste d'atterrissage au nord-ouest de Santo à Lajmol (carte 1), sur laquelle un avion Islander de huit places se pose une fois par semaine, ce petit aéroport régional n'est fréquenté qu'exceptionnellement par les populations locales. Ce sont surtout des exploiteurs forestiers, plus rarement des agents du gouvernement (forces de l'ordre et hommes politiques) et les instituteurs de la région qui sont à même de pouvoir profiter de ce moyen de transport. En l'absence de routes et de moyens financiers suffisants, les habitants se contentent des hors-bord ou, le plus souvent, circulent à pied ou à cheval.

Situé sur le littoral nord-ouest de l'île de Santo, à quelques kilomètres au sud de cet aéroport, le village de Penaoru est composé de trois hameaux principaux comprenant en tout une vingtaine de ménages. Là comme ailleurs dans la région, chacun vit de l'agriculture de subsistance (jardins d'ignames et de taros), de l'élevage (vaches, cochons, poules), de la pêche et de la chasse ; et chacun tire de modestes et occasionnels revenus des cultures de rente locales (coprah, cacao, kava et bois de santal) qu'un 
bateau collecteur vient chercher d'une à trois fois par an. Avec un revenu moyen annuel par famille de $350 €$, il est souvent difficile de subvenir aux besoins de première nécessité : la scolarité des enfants (autour de $150 €$ par an et par enfant), un déplacement en hors-bord (souvent payé à crédit) ou quelques biens manufacturés (vêtements, riz, sel, sucre, kérosène pour les lampestempête, allumettes, essence pour les bateaux). À Penaoru, il n'y a pas de système de télécommunication ni d'approvisionnement en eau et le dispensaire le plus proche se trouve à quatre heures de marche de là, plus au nord, à Nokuku.

\section{Le déroulement des négociations}

Pour ces habitants de la côte ouest de Santo, l'aventure Santo 2006 commença en décembre 2005 lorsqu'une première délégation vint repérer les lieux et choisir les sites où les scientifiques de l'expédition allaient s'établir et effectuer leurs recherches. Parmi ces sites, l'un d'eux devait servir de camp de base pour abriter les nombreux participants au « module FMR » ainsi que l'arboglisseur.

«La première fois qu'ils sont venus, en décembre 2005 , c'était pour voir si notre environnement convenait à leurs recherches et pour trouver un lieu pour construire leur camp. En repartant, ils nous ont dit qu'ils n'étaient pas encore décidés et nous enverraient une lettre pour nous informer de leur choix. Janvier est arrivé, puis février et mars... et aucune lettre ne nous est parvenue. C'est en avril 2006 qu'ils sont arrivés par bateau, sans prévenir. On est allés sur le site du camp et on a marqué les emplacements des maisons. » (Roni, février 2007, Penaoru).

Au tout début de l'année 2006, quelques jours après les premiers repérages, un événement singulier se produisit à Penaoru, qui fut immédiatement associé à la venue imminente des scientifiques sur la côte ouest. Un pan entier de la montagne sacrée Ate At' (Dieu en langue locale), dressée au-dessus du chemin menant au camp, s'effondra, laissant visible un morceau de roche brute d'un blanc éclatant, l'annonce de quelque chose... mais de quoi ? Dans un premier temps, les villageois de la région interprétèrent cet événement comme le signe de très grandes découvertes scientifiques à venir et de changements bénéfiques pour eux. Mais plusieurs mois après le départ des scientifiques, les choses paraissaient moins claires dans leurs esprits. Certains associèrent le creux formé dans la roche au vide laissé par cet éphémère tourbillon occidental, tandis que d'autres, en colère, interprétèrent ce drame comme la preuve irréfutable selon laquelle les membres de cette expédition, pour une raison inconnue, avaient volontairement endommagé un site sacré.

\section{La construction du camp}

Lors de la visite réalisée en avril 2006, il fut décidé de retenir comme sites de recherche pour ce module les alentours du Mont Tabwemasana et de sa grande rivière Pua et les villages littoraux de Tasmate (côte ouest) et de Peavot (Big Bay) en raison des diverses niches écologiques qui y étaient présentes (carte 1). Mais ce fut le site de Sevrapek, à $4 \mathrm{~km}$ de Penaoru, qui fut choisi pour établir le camp de base des chercheurs et faire fonctionner l'aérostat. Un conseil villageois fut mis en place pour l'occasion : il comprenait une dizaine d'habitants de Penaoru au nombre desquels le chef Wartut, le représentant religieux Rupen et le représentant local de la province regroupant les îles Santo et Malo (appelée SANMA), Josen. Ce conseil donna son accord pour que le camp de base des scientifiques soit construit à Sevrapek, une terre exempte de litiges fonciers car commune à tous les clans fondateurs de cette région. C'est à ce moment-là que fut estimé le coût de la construction des infrastructures et des aménagements nécessaires à l'expédition et que furent fixés les salaires des travailleurs locaux, qui n'étaient pas en mesure de deviner ce que seraient les tâches à accomplir et auxquels peu d'informations concrètes furent alors données. De l'avis de Josen :

« Ce contrat n'était pas vraiment un contrat. [...] il a surtout été question de la construction des maisons, de ce qu'ils voulaient que l'on fasse, de ce qu'ils attendaient de nous. Mais il n'a pas été question de nos attentes à nous, de ce que nous voulions. Il n'y avait pas la place pour ça. » (Josen, Penaoru, février 2007)

Localisé à 119 mètres d'altitude, sur les berges de la rivière éponyme, Sevrapek était un coin de forêt couvert de végétation de repousse dont l'aspect du sol laissait deviner d'anciens aménagements pour jardins irrigués. Abandonné depuis des décennies, ce site fut entièrement réaménagé par les villageois pour la venue des membres de l'expédition.

Dans les mois qui précédèrent l'arrivée des scientifiques, et pour la somme globale de 500000 vatus (environ $3800 €)^{18}$, les habitants

18. C'est une réunion villageoise qui permit de décider de l'utilisation de cette somme : achever la construction « en dur » (murs et sol cimentés, toit en tôle ondulée) de l'église de Penaoru - c'est-à-dire se donner les moyens de posséder une église 
de Penaoru et des villages alentour (Hokua, Olpoi, Wunon, Nokuku, Penel, Pwanpwanon, Petawat, Sulesay et Wunavay) ont amélioré, en déplaçant des pierres et en débroussaillant, les quatre kilomètres de chemin qui séparaient le camp de base du bord de mer où fut construite une maison de bambou pour stocker le matériel de l'expédition. Ils ont aménagé le site du dirigeable, en dépierrant et en aplanissant le sol sur une surface de $26 \mathrm{~m}^{2}$. Ils ont dû également construire un pont de bambous sur le dernier bras de la rivière Penaoru pour permettre un accès plus aisé au camp. Ils ont bâti huit maisons, une cuisine, deux laboratoires, deux abris pour les générateurs et une salle de convivialité (localement appelée Nakamal). Ils ont dû également creuser profondément le sol pour aménager deux latrines. Les habitants des villages de la côte nord-ouest de Santo n'ont pas ménagé leur peine et leur investissement dans ce projet. Hormis les quelques pelles, la brouette et les bâches de plastique qui leur furent données par les organisateurs du projet, les matériaux de construction utilisés (bois, bambou, feuilles de Metroxylon pour la fabrication des tuiles végétales, lianes) furent collectés dans leur forêt, transportés à dos d'homme et taillés avec leurs propres haches et machettes.

À l'arrivée des premiers participants de la mission, fin octobre 2006, les éléments indispensables au travail et au confort des chercheurs furent installés. L'électricité, un système de pompage de l'eau de la rivière Penaoru, des congélateurs, une gazinière, un téléphone-satellite et une connexion à internet firent ainsi leur apparition dans ce village qui n'avait évidemment jamais connu rien de tel. On l'a dit, il ne faut pas pour autant imaginer que les habitants de la côte ouest, dont les seuls contacts avec la vie à l'occidentale ont lieu lorsqu'ils se rendent en ville, vivent hors du monde. De leur recoin d'île, ils sont informés, grâce à la radio, des événements nationaux et internationaux. Ils sont aussi pleinement membres de leur État, sont impliqués depuis longtemps dans l'économie de marché et dans la vie politique de leur pays. Certains des villageois de cette région obtiennent occasionnellement de petits contrats de travail en ville, travaillent pour les exploitants forestiers de la région ou, comme Josen, se rendent régulièrement en ville où ils ont des responsabilités politiques. Aussi, certains des agissements des membres de l'expédition (considération, salaires pratiqués, nature des charges à transporter, etc.) furent-ils mal perçus, et beaucoup de villageois estimèrent-ils avoir été exploités comme du temps du condominium... Souvent comparée dans la presse aux grandes expéditions naturalistes des siècles passés, avec évidemment des moyens techniques résolument modernes et l'ambition d'un cadre éthique irréprochable, ces réflexions et les plaintes que j'ai souvent entendues lors de mes enquêtes et du tournage du documentaire nous conduisent à poser la question suivante : l'expédition Santo 2006 s'est-t-elle réellement émancipée de l'état d'esprit de cette époque ?

\section{Perception locale de l'événement et désertion du camp}

Dès les premiers jours de novembre 2006, Sevrapek devint un lieu de vie concentrant une inhabituelle effervescence humaine et offrant une technologie et des services inédits dans la région. C'est par ce campement que les nombreux scientifiques du « module FMR » ont transité pour des durées allant d'une semaine à un mois. Employés durant la mission en tant que porteurs, guides, lavandières et cuisinières, des dizaines d'habitants de la région s'y sont rendus quotidiennement lorsqu'ils résidaient à proximité, ou y ont séjourné le temps de la présence des membres de l'expédition lorsque leur village d'origine était plus éloigné.

Tous furent rémunérés " à la tâche », payée de 300 à 400 vatus (soit 2 à $3 €$; photo 3 ). Qu'il s'agisse du portage, à dos d'homme et sur $4 \mathrm{~km}$, du matériel scientifique et des affaires personnelles des chercheurs, des stocks de nourriture " sèche ", ou du transport des congélateurs, des générateurs, des différents éléments de l'arboglisseur et de son dôme, des dizaines de bouteilles de gaz et d'hélium (pesant entre 80 et $110 \mathrm{~kg}$ chacune ; photos 4 et 5) nécessaires à son fonctionnement (mais aussi au séchage des spécimens biologiques et à la cuisine), la somme reçue ne variait guère. Les guides étaient, eux, mieux payés (1 000 vatus la journée, soit à peu près $7 €)$, car leur travail impliquait aussi le débroussaillage d'anciens chemins et le portage de certains produits (riz, conserves, eau potable, presses à herbiers, matériel de camping...). Les villageois ont eu par ailleurs l'opportunité de vendre un grand nombre de

« moderne » (mais aussi durable) identique à celles que l'on peut voir dans la plupart des villages de cette région depuis une quinzaine d'années. Ce choix laissa les responsables du « module FMR» perplexes car ils jugèrent cette décision absurde au vu des besoins matériels locaux. Sans entrer ici dans les détails, précisons que, pour les habitants très pratiquants de cette région christianisée depuis la fin du XIX ${ }^{\mathrm{e}}$, posséder une telle église est autant un gage de protection contre les attaques de sorcellerie que l'on redoute encore, qu'un signe extérieur de richesse (matérielle et spirituelle) lors de la venue de visiteurs (parents ou étrangers). 


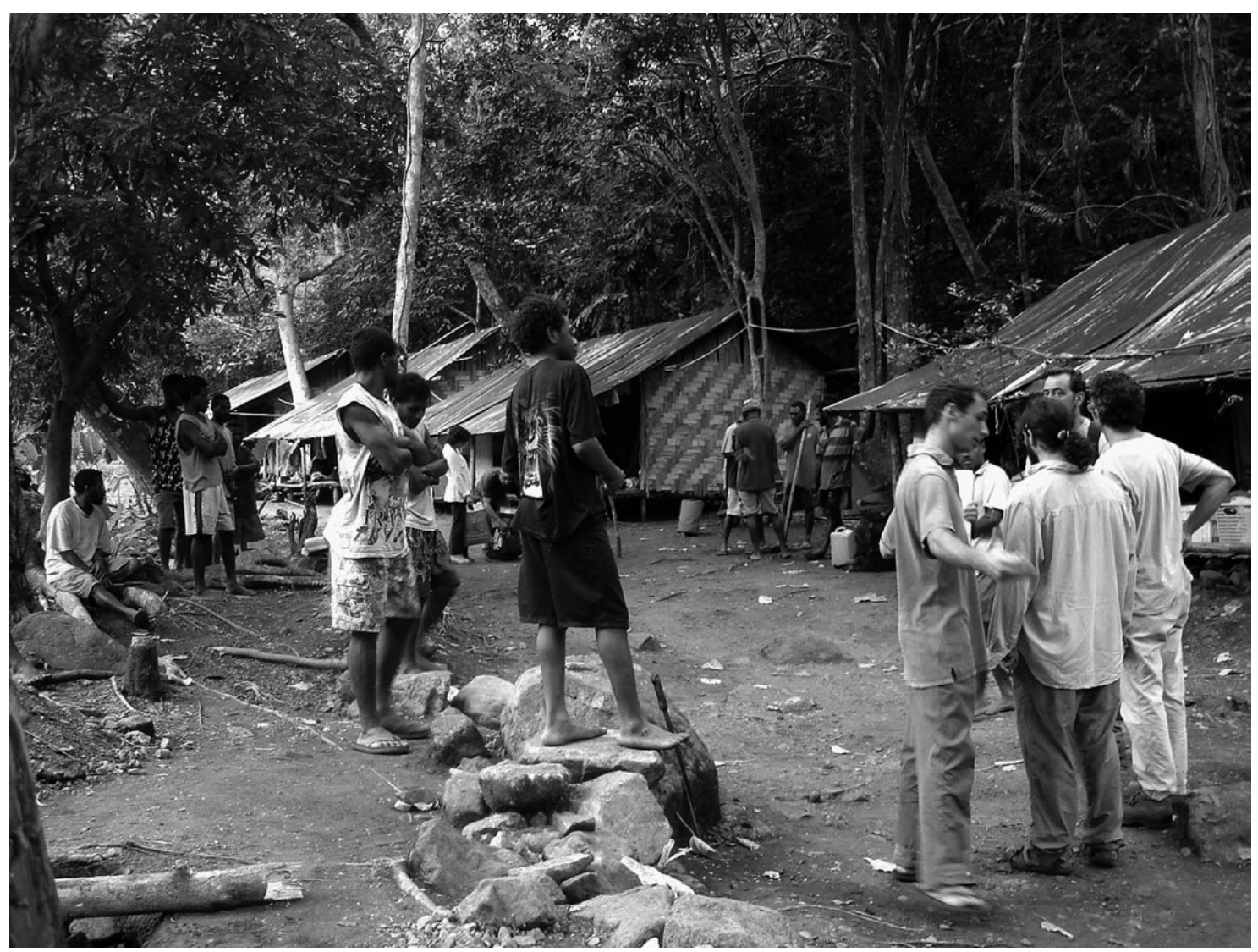

Рното 3. - Le camp de Sevrapek : porteurs et guides attendent de savoir qui part en forêt et avec quelle charge (cliché de l'auteur)

leurs cochons, vaches, poules ainsi que divers produits de leurs jardins (fruits, noix de coco, tubercules), mais sans toujours réaliser que le fort appétit de leurs invités grèverait à ce point l'état de leurs réserves. Le transport de ces vivres se faisait en pirogue jusqu'à Penaoru (lorsque ces aliments provenaient des villages alentour), puis à pied ou à cheval du bord de mer jusqu'au camp.

Le mot city qu'ont apposé les habitants de Penaoru au nom du camp Sevrapek en dit long sur ce que ce village éphémère a représenté à leurs yeux. De l'avis de tous, ce camp, situé à seulement une heure de marche de chez eux, a possédé durant un mois toutes les apparences d'une ville. Les gens s'y rendaient pour chercher du travail, « Noirs » et « Blancs » s'y côtoyaient quotidiennement et les habitants de la côte ouest trouvaient là certains des attraits et des services de la vie urbaine, dont ils ne bénéficient pas et dont ils ont finalement assez peu profité : l'électricité, le téléphone, l'eau, une infirmerie, internet, de l'alcool et des cigarettes. Quant au site où se trouvait l'arbodôme, il est devenu un lieu incontournable où les villageois de toute la région de l'Ouest de Santo sont venus guetter, sans relâche, des heures et des jours durant, l'envol de cette nouvelle version du Radeau des Cimes.

Pour les habitants de la région, le séjour de l'expédition Santo 2006 a été, à bien des égards, exceptionnel : sur une durée aussi courte et «à domicile », on n'avait jamais vu autant de «Blancs» et on n'avait jamais gagné autant d'argent ${ }^{19}$. On n'avait également jamais délaissé à ce point le travail agricole, ce qui causa bien des soucis et entraîna de longues journées de travail après le départ des scientifiques pour remettre en état les jardins vivriers et les plantations de coprah.

19. En un mois, les habitants de Penaoru ayant vendu régulièrement les produits de leurs jardins ou de leurs élevages ont obtenu entre 20000 et 30000 vatus (soit entre 150 et $230 €$ ). Cet argent a permis de payer les frais de scolarité de l'un de leurs enfants pour l'année. Ceux employés comme porteurs et guides ont en moyenne reçu 10000 vatus (environ $75 €$ ), tandis que d'autres, originaires de villages voisins et ayant travaillé plus occasionnellement, ont perçu entre 2000 et 8000 vatus (soit entre 15 et $60 €$ ). Cet argent a principalement servi à s'acquitter de dettes, à payer un déplacement en ville ou à acquérir quelques biens manufacturés. 

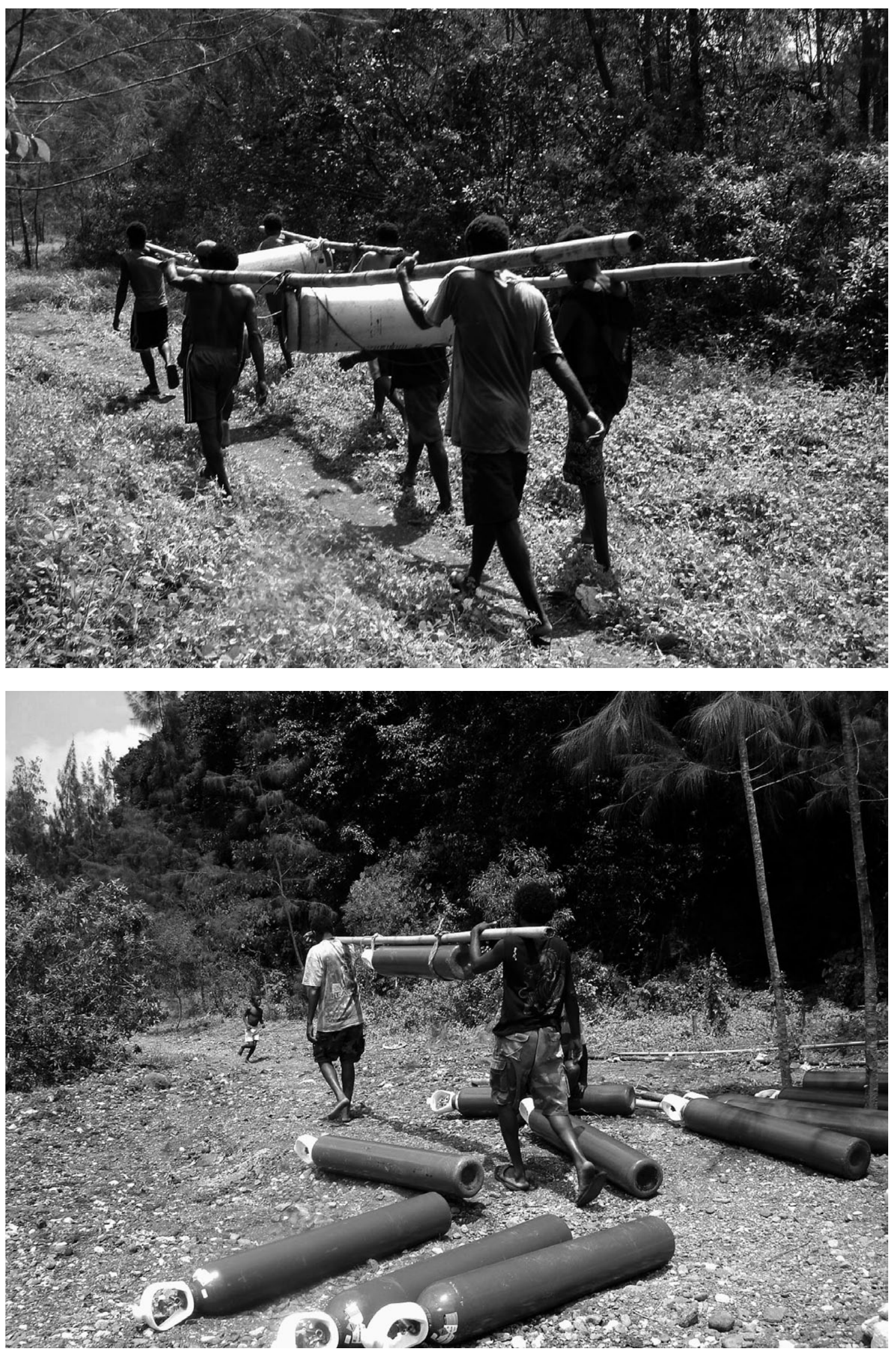

Photos 4 et 5. - Transport des bouteilles de gaz et d'hélium du site de l'arboglisseur au bord de mer (clichés de l'auteur)

À l'aide de bambous, les bouteilles de gaz comme celles d'hélium sont transportées « à dos d'homme » sur les 4 km qui séparent le camp du bord de mer. 
Mais, au-delà d'un lieu où l'on s'est rendu pour gagner de l'argent, Sevrapek est devenu une sorte de village de «Blancs » reconstitué, un endroit où les habitants de la région sont venus observer à loisir un spectacle d'un genre nouveau. Un village factice qui, d'une certaine façon et en forçant le trait, a ressemblé à une forme inversée d'exposition coloniale.

De tous les modules du projet Santo 2006, celui dénommé « Forêts, Montagnes, Rivières » concentra le plus grand nombre de scientifiques. C'est dans le camp de Sevrapek qui lui servit de base que les contacts entre les chercheurs, les organisateurs du projet et les ni-Vanuatu furent les plus intenses. Il n'est donc nullement surprenant qu'ils aient été aussi les plus problématiques.

Dans ce contexte, une grève éclata à la mi-novembre 2006 en raison d'un mécontentement croissant relatif aux salaires pratiqués ${ }^{20}$. Car, si un accord avait bien été signé avant le début du travail des scientifiques à Penaoru, personne sur place n'avait alors la moindre idée de la nature des objets qu'il faudrait transporter. Les villageois réclamèrent par ailleurs des contreparties matérielles durables et nécessaires à leur quotidien (en l'occurrence un système d'approvisionnement en eau et un téléphone) en présentant, à la fin novembre, une pétition lors de la visite d'une délégation officielle composée de ministres du Vanuatu et de représentants des autorités françaises ${ }^{21}$. Ces demandes n'ont pas été satisfaites et il est désormais évident pour eux que si un projet de recherche similaire venait à leur être présenté, le contrat serait négocié différemment qu'il ne l'avait été ici ${ }^{22}$.

Si de l'avis de certains (voir l'article de Elsa Faugère, dans ce même numéro) les contreparties financières sont loin d'avoir été négligeables compte tenu du contexte local plutôt peu monétarisé, elles ont en tout cas été jugées insatisfaisantes, car non durables, par les popu- lations locales. Car, concrètement, que restait-il à Penaoru après le départ des scientifiques hormis quelques bouteilles de verre vides utilisées pour stocker de l'eau potable au village, des containers en plastique pour collecter de l'eau de pluie, un bidon de 80 litres de kérosène, des câbles électriques, des bâches de plastique, quelques tréteaux, des matelas et des planches de bois ? Force est de constater que les biens matériels laissés par l'expédition sont bien mal adaptés aux besoins réels des habitants de la région. Ces derniers attendaient plus de générosité et d'investissement en termes de moyens de communication et de services de santé de la part d'un projet dont ils n'ont évalué la réelle envergure qu'une fois les scientifiques sur place et pour lequel ils se sont eux-mêmes beaucoup impliqués en mettant à disposition leurs terres, en fournissant un travail difficile et en délaissant leurs jardins.

« J'ai fait des commentaires négatifs quand j'ai lu la convention. Ça me semblait insatisfaisant pour les gens du Vanuatu [...]. Ça ne servait à rien, les rapports, les productions académiques par exemple pour les gens de la côte ouest [de Santo] [...]. Il n'y avait aucun bénéfice pour les communautés locales [...]. L'argent laissé dans les communautés, c'est bien, mais il aurait fallu que soit produit du matériel pour les écoles primaires ou qu'ils fassent un film éducatif en bislama [...] [c'est-à-dire] des choses accessibles pour les gens. Les habitants de Penaoru avaient besoin d'un système d'approvisionnement en eau, d'un téléphone. Ça a été clairement demandé lors de la venue des officiels au camp [...] Ça aurait dû être dans le projet [...]. Et ça aurait été suffisant comme retour pour les gens [...]. » (Raph Regenvanu, directeur du Vanuatu National Cultural Council, mars 2007, Port-Vila)

Les populations locales ont aussi regretté l'absence de participation d'enquêteurs de terrain (fieldworkers) du Centre culturel $\mathrm{du}$ Vanuatu dont le travail aurait pu favoriser une meilleure compréhension des objectifs de l'expédition Santo 2006. La plupart des gens de la côte

20. Les villageois réclamaient une augmentation de 2 à $4 €$ par tâche. La réponse fut, en substance, la suivante : « il est trop tard pour changer les salaires... et le budget est serré ».

21. Ayant fait l'objet de l'important déploiement médiatique que l'on sait, Sevrapek attira un grand nombre de visiteurs étrangers à la région : des ministres ni-vanuatu, le directeur du Vanuatu National Cultural Council, un représentant de Radio Vanuatu, des membres de l'Ambassade de France au Vanuatu, un représentant du Fonds Pacifique et des sponsors de l'expédition.

22. Lors de la venue des sponsors (Solvay-Solvin, Pro-Natura et Ricoh), le 26 novembre, le président de Pro-Natura (se souvenant bien à propos de l'ambition de son ONG de « répondre aux problèmes socio-économiques et environnementaux des communautés rurales des pays du sud ») et celui de Solvay m'assurèrent de leur souci de « laisser quelque chose de durable » après Santo 2006. À leur demande, j'ai donc rédigé un rapport expliquant les raisons pour lesquelles les gens de Penaoru avait notamment besoin d'un système d'adduction en eau. Ce rapport incluait mes observations ainsi que les données collectées sur place par Philippe Keith du MNHN (comparaison de la diversité de la faune d'eau douce en altitude et en bord de mer ; évolution de la température de l'eau $-21^{\circ}$ à 600 mètres d'altitude, $23^{\circ}$ à $119 \mathrm{~m}$, soit au camp, et jusqu'à $36^{\circ}$ en bord de mer, c'est-à-dire là où les gens se baignent et collectent de l'eau pour cuisiner ou boire) et par le médecin de l'équipe médicale du camp, Cyril Chevalier, qui avait identifié plusieurs problèmes récurrents de santé (diarrhées, troubles digestifs, infections cutanées). Depuis, le directeur de Pro-Natura soutient qu'il n'est pas habilité à suivre techniquement ce type de projet; quant au directeur de Solvay, a priori tout désigné à assurer cette tâche, il reste depuis près de deux ans muet à mes multiples courriels. 
ouest de l'île se demandent par exemple à quoi le travail des biologistes va servir.

Bien que consulté en amont lors de la préparation de l'expédition, le Centre culturel a, quant à lui, déploré d'avoir été négligé pendant le déroulement de la mission. Pour l'actuel directeur du VNCC, « une fois que le projet a commencé, on n'a plus eu de relations avec les organisateurs du projet... et aucun fieldworker n'a été employé » (Raph Regenvanu, mars 2007, PortVila). Par ailleurs, et bien que cela soit clairement formulé dans le Cultural Research Policy, le directeur du Centre culturel du Vanuatu, Marcellin Abong, a regretté que les rushes (la totalité des images) du reportage pour l'émission de télévision Thalassa n'aient jamais été remis au National Film and Sound Unit du Centre Culturel $^{23}$. Quant au contenu de ce reportage, comme son titre évocateur le laisse présager (Les aventuriers de l'île planète), il fait uniquement état $\mathrm{du}$ travail de quelques naturalistes de l'expédition, misant tout sur l'extrême photogénie des mollusques marins et des insectes tropicaux et ne laissant apparaître les habitants du Vanuatu qu'à la manière des sept nains de Blanche Neige (en arrière-plan, le sourire aux lèvres, portant des cartons) ou en montrant les images, saisissantes et spectaculaires il est vrai, du débarquement de la barge sur la plage de Penaoru (un accueil rappelant sans doute celui fait au XIX ${ }^{\mathrm{e}}$ siècle aux grands voyageurs, comme le dit, émerveillé, l'un des membres de l'expédition interrogé pour l'occasion).

Certes, les gens de la côte ouest de Santo tirèrent un enseignement de cette expérience : ils aimeraient que, dans le cas où un nouveau projet du même genre s'élaborerait, leur fût exposée de façon précise la nature du travail qu'ils auraient à fournir et expliquée la finalité des recherches des scientifiques afin qu'une discussion à égalité puisse s'engager à propos des modalités de leur participation. Comme le dit Josen :

« La prochaine fois, il faudra qu'ils soient satisfaits, mais nous aussi nous devrons l'être [...]. Nous aurons notre mot à dire lors de la rédaction du contrat et nous devrons être entendus. Notre dos est dur maintenant, ça ne se passera plus comme ça. » (Josen, Penaoru, février 2007)

Autrement dit, les villageois aimeraient être considérés comme des participants à un projet collectif et non comme de simples exécutants auxquels il n'y aurait nul besoin de fournir d'explications pour qu'ils accomplissent leurs tâches.

\section{Conclusion}

Au-delà du seul cas de Santo 2006, ces critiques pointent du doigt la complexité du regard des occidentaux sur l'Autre, du rapport de la France à l'une de ses anciennes colonies et de celle, quelque peu hypocrite, du «partage des avantages » avec les populations locales. Certains doutent qu'il soit légitime de demander à une expédition naturaliste et à ses acteurs de faire également un travail de développement local et un travail social (voir article d'Elsa Faugère dans ce même numéro). Ils s'interrogent aussi sur ce que cette expédition a coûté au Vanuatu et aux populations locales, ou si elles y ont finalement perdu quelque chose.

Malheureusement, cette expédition a bel et bien coûté aux populations locales, et ce tant en termes de travail et d'attentes (déçues) que de déstabilisation du système de subsistance même des villages. Elle a aussi eu un coût, au sens propre, pour le pays puisque l'ensemble des dépenses liées à Santo 2006 ont été détaxées et n'ont donc rien rapporté aux institutions publiques de l'un des pays les plus pauvres de la planète.

Mais exprimons les choses autrement : si un richissime étranger s'introduisait chez vous, s'y installait et vous demandait de porter ses effets, de coopérer servilement et aveuglément à ses projets quels qu'ils soient, se servant dans votre jardin ou piochant dans vos placards à la mesure de son gros appétit, tout en ne vous offrant qu'un maigre pourboire et en affirmant que cela ne vous coûte pas grand-chose, vous protesteriez probablement, pour des raisons d'équité, de réciprocité dans l'échange et de respect de votre personne. Dans le cas de Santo 2006, le problème trouve bien son origine dans un déséquilibre entre les services rendus et le retour financier auquel s'est parfois greffé un manque de respect des organisateurs du projet vis-à-vis des populations locales et des institutions du Vanuatu. On peut se poser la question de savoir pourquoi les dirigeants d'une mission disposant d'un budget de 1,2 millions d'euros ont décidé de ne pas accéder aux modestes revendications salariales des travailleurs de Penaoru. Comment justifier ce refus alors qu'ils rémunéraient généreusement (autour de $2500 €$ par mois) un certain expatrié franco-espagnol employé comme coordinateur local du projet, tandis que son assistant, un ni-Vanuatu, percevait à peine l'équivalent du RMI français (environ $400 €$ )?

23. Disposant d'un budget d'environ $750000 €$, ATOM Productions ne s'est jamais acquittée des « droits » demandés par le Centre culturel (soit le dépôt d'une caution de $760 €$ et le versement d'un « droit de filmer » de $760 €$ ). 
Mais qui dit échange et équité sous-entend rapport social. Le problème soulevé par cette mission tient précisément dans la nature des relations établies avec les "partenaires locaux», finalement négligés, ce au sein d'une expédition présentée en France comme remplissant une noble mission au bénéfice de la connaissance et de la science. Or, ces relations ont été du même type que celles qu'aurait mises en place une multinationale faisant de l'exploitation forestière ou collectant des spécimens biologiques à des fins marchandes. Alors qu'aucune pression concurrentielle ni aucun environnement industriel ne les y forçait, les organisateurs ont exploité au maximum la main-d'œuvre locale, puisé dans les richesses biologiques du pays (bien qu'à des fins académiques) et se sont procuré biens et services à bas prix ${ }^{24}$. Essayer de comprendre les raisons sous-tendant de tels agissements permet de révéler quelque chose d'essentiel concernant la nature même du rapport Nord-Sud d'aujourd'hui. Car, dans ce cas précis, l'exploitation abusive de la main-d'œuvre n'est-elle pas doublée d'une discrimination de type racial débouchant finalement sur la question de la considération de l'Autre ? Cela ne met-il pas au jour un rapport social établi de longue date selon des schémas préconçus se reproduisant presque systématiquement dès lors que l'action se situe dans un ancien pays colonisé ou dans un pays pauvre? Le Vanuatu est certes indépendant, mais les comportements et les rapports sociaux ne restent-ils pas finalement identiques à ceux qui prédominaient durant les siècles précédents ou pendant la colonisation?

\section{BIBLIOGRAPHIE}

Barrais Delphine, 2007. Santo 2006, une expédition scientifique d'exception, rapport de l'Ambassade de France au Vanuatu (http://www.ambafrance.si/ spip.php?article675).

Bouchet Philippe, Bruno Corbara, Hervé Le GuyaDER et Rufino PINEDA, 2006. Santo 2006 : La diversité dans tous ses états, du battant des lames au sommet des montagnes, Paris, IRD/MNHN/ProNatura.

Bouchet Philippe, Hervé Le Guyader et Olivier PasCAL (eds), 2007. Santo 2006 Expedition. Progress Report, Paris, IRD/MNHN/Pro-Natura.

Collomb Étienne, 2007. Les Indiana Jones de la biodiversité, Géo 338, pp. 122-134.

Lescroart Marie, 2007. Santo 2006, Grand inventaire dans l'île aux trésors, Terre Sauvage 225, pp. 12-35.

Morin Hervé, 2006 (3 juillet). Une expédition internationale va explorer la biodiversité du Vanuatu, Le Monde.

Royson Willie, 2006 (28 June). Scientific research gets first butting from government institution, Daily Post.

Schmitr Matthias, 2007. L'île laboratoire, vSD 1534, pp. 46-53.

TARDIEU Vincent et Lise BARNÉOUd, 2007. Santo. Les explorateurs de l'île Planète, Belin.

Site officiel du Vanuatu Cultural Centre: www. vanuatuculture.org/research/050520_culturalre searchpolicy.html

Site officiel de l'expédition Santo 2006 : www. Santo2006.org

Site officiel de Pro-Natura International : www.pronatura.org

24. Je fais ici allusion aux détaxes mentionnées dans la convention, mais aussi à une quinzaine de pierres de haches qui ont été « échangées » à Penaoru contre des lampes torches à dynamo, au grand dam d'Aldi Ezekiel, le fieldworker qui nous assista en qualité d'ingénieur du son lors du tournage de Sevrapek City quelques mois plus tard. 